\title{
Model-theoretic consequences of a theorem of Campana and Fujiki
}

by

\author{
Anand Pillay (Urbana, IL, and Berlin)
}

\begin{abstract}
We give a model-theoretic interpretation of a result by Campana and Fujiki on the algebraicity of certain spaces of cycles on compact complex spaces. The model-theoretic interpretation is in the language of canonical bases, and says that if $b, c$ are tuples in an elementary extension $\mathcal{A}^{*}$ of the structure $\mathcal{A}$ of compact complex manifolds, and $b$ is the canonical base of $\operatorname{tp}(c / b)$, then $\operatorname{tp}(b / c)$ is internal to the sort $\left(\mathbb{P}^{1}\right)^{*}$. The Zilber dichotomy in $\mathcal{A}^{*}$ follows immediately (a type of $U$-rank 1 is locally modular or nonorthogonal to the field $\mathbb{C}^{*}$ ), as well as the "algebraicity" of any subvariety $X$ of a group $G$ definable in $\mathcal{A}^{*}$ such that $\operatorname{Stab}(X)$ is trivial.
\end{abstract}

1. Introduction. This paper concerns the interaction between complex-geometric notions and model-theoretic notions in the structure theory of compact complex spaces. It has been known for some time that modeltheoretic ideas yield a rather striking dichotomy for simple compact complex manifolds $M$ : either $M$ is algebraic, or else there is no "2-parameter" family of finite-to-finite analytic correspondences between $M$ and itself. But, up to now, the only proof of this of which I was aware went through the results on Zariski geometries and their validity for compact complex manifolds, together with some other ingredients (see [6], [7] and [11]). It turns out that the dichotomy above and more are almost immediate consequences of a theorem proved independently by Campana [1] and Fujiki [3]. They prove, roughly speaking, that if $S$ is a compact space of cycles $\left(Z_{s}: s \in S\right)$ on a compact complex space $X$ then the natural morphism from the graph $\left.\left(\{x, s): x \in Z_{s}, s \in S\right\}\right)$ of $S$ to $X$ is a Moishezon map. Via a translation established by Moosa ([9], [10]), this yields the following striking statement in the language of canonical bases (to be read in a saturated elementary extension $\mathcal{A}^{*}$ of the many-sorted structure $\mathcal{A}$ of compact complex spaces):

$(*) \quad$ for any $b, c, \operatorname{tp}(\mathrm{Cb}(\operatorname{tp}(c / b)) / c)$ is "algebraic", that is, internal to $\mathbb{C}^{*}$.

2000 Mathematics Subject Classification: 03C45, 32J27.

Partially supported by an NSF grant and a Humboldt Foundation Research Award. 
The statement $(*)$ (or the original Campana-Fujiki statement) yields the dichotomy for $U$-rank 1 types in $\mathcal{A}^{*}$ : they are modular or "algebraic". Also the algebraicity of suitable subvarieties of meromorphic groups and homogeneous spaces follows directly, subsuming results of Ueno [15] as well as results from [8]. I guess that the benefit of the model-theoretic translation (*) lies in being able to work directly with bimeromorphic objects without worrying about specific compactifications. As the reader might surmise, the statement $(*)$, when suitably re-interpreted, is also directly provable (using suitable jet spaces) in other algebraic/model-theoretic structures of interest, such as differential and difference fields. (See [14].)

The details of the observations above are given in the next section.

2. Results. For the theory of compact complex spaces see [4]. For the model-theoretic treatment of this subject see [11].

Let us work towards stating the Campana-Fujiki theorem. Let $X$ be a reduced, irreducible, compact complex space. There are two notions of cycle spaces on $X$. The Douady space $D(X)$ parametrizes pure-dimensional analytic subsets of $X$. The Barlet space $C(X)$ parametrizes integral linear combinations of irreducible analytic subsets (of the same dimension) of $X$. Campana works with Barlet spaces and Fujiki with Douady spaces. A morphism $f: Y \rightarrow X$ of compact complex spaces is said to be projective if there is a coherent analytic sheaf $\mathcal{F}$ over $X$ and an embedding (over $X$ ) $h$ of $Y$ into the projective linear space $\mathcal{P}(\mathcal{F})$ over $X$ associated with $\mathcal{F}$ such that $\pi \circ h=f$, where $\pi: \mathcal{P}(\mathcal{F}) \rightarrow X$ is the map realizing $\mathcal{P}(\mathcal{F})$ as a fibre space over $X$. A morphism $f: Y \rightarrow X$ is said to be Moishezon if it is bimeromorphic (over $X$ ) to a projective morphism $f^{\prime}: Y^{\prime} \rightarrow X$. Campana proves:

FACT 2.1. Let $S$ be an irreducible, compact, analytic subset of $C(X)$. Let $Z_{s}$ denote the cycle parameterized by $s \in S$. Assume that for general $s \in S, Z_{s}$ is irreducible. Let $Y=\left\{(x, s) \in X \times S: x \in Z_{s}, s \in S\right\}$, and let $f: Y \rightarrow X$ be the projection to the first coordinate. Then $f$ is a Moishezon map.

In fact the above statement comes from [2] (Theorem 3.6). The original theorem in [1] states that $f$ factors through an embedding in a suitable Grassmannian of $\mathcal{F}$ over $X$, where $\mathcal{F}$ is the coherent analytic sheaf of germs of differential operators of suitably bounded order. Such an $f$ is Moishezon. In Fujiki's statement, $C(X)$ is replaced by $D(X)$.

We now work towards the model-theoretic interpretation. $\mathcal{A}$ is the manysorted structure of compact complex spaces $X_{i}$ with predicates for analytic subvarieties of Cartesian products $X_{i_{1}} \times \ldots \times X_{i_{n}}$. Note that with this "language", all elements of all sorts in $\mathcal{A}$ are named by constants. $\operatorname{Th}(\mathcal{A})$ has 
quantifier elimination, elimination of imaginaries and is stable with finite Morley rank (sort-by-sort). $\mathcal{A}^{*}$ will be a very saturated elementary extension of $\mathcal{A}$. Among the sorts in $\mathcal{A}$ is the projective line $\mathbb{P}_{1}$ over $\mathbb{C}$. There is no harm (via Chow's theorem) in identifying this sort with the complex field $\mathbb{C}$ equipped with addition, multiplication, and constants for all elements (they are bi-interpretable). $\mathbb{C}^{*}$ denotes the "extension" of this sort in $\mathcal{A}^{*}$. Let $b, c$ be tuples from $\mathcal{A}^{*}$. Following [9], [10], we will say that $\operatorname{tp}(c / b)$ is Moishezon if $\operatorname{tp}(c / \operatorname{acl}(b))$ is internal to $\mathbb{C}^{*}$, or equivalently if there is some finite tuple $b^{\prime}$ including $b$ and independent of $c$ over $b$ such that $c \in \operatorname{dcl}\left(d, b^{\prime}\right)$ for some tuple $d$ from $\mathbb{C}^{*}$. (Note that $\operatorname{tp}(c / b)$ is Moishezon just if every stationarization of it is Moishezon.) Moosa [10] observes the following:

FACT 2.2. Suppose that $X, Y$ are irreducible compact complex spaces and that $f: Y \rightarrow X$ is a Moishezon map. Let $c \in Y^{*}$ be a generic point of $Y$ $($ over $\mathcal{A})$. Then $\operatorname{tp}(c / f(c))$ is Moishezon.

We can now obtain:

Theorem 2.3. Let $b, c$ be finite tuples from $\mathcal{A}^{*}$. Assume that $\operatorname{tp}(c / b)$ is stationary and that $b=\mathrm{Cb}(\operatorname{tp}(c / b))$. Then $\operatorname{tp}(b / c)$ is Moishezon.

Proof. Let $X, S, Z$ be irreducible compact complex spaces of which $c, b$ and $(c, b)$ respectively are generic points (over $\mathcal{A}$ ). Then $Z_{b}$ is irreducible with generic point $c$, and moreover $b$ is a canonical parameter for $Z_{b}$ (by quantifier elimination). Replacing $S$ by a suitable modification, we may assume that the projection $\pi: Z \rightarrow S$ is flat. The universal properties of the Douady space $D(X)$ of $X$ yield a morphism $p: S \rightarrow D(X)$ such that $p(s)$ corresponds to $Z_{s}$. Then $p(S)$ is a compact irreducible analytic subset of $D(X)$. Also, by compactness, there is a Zariski open subset $U$ of $S$ such that for $s_{1}, s_{2} \in U$, $Z_{s_{1}}=Z_{s_{2}}$ iff $s_{1}=s_{2}$. Thus $p: S \rightarrow p(S)$ is a modification. The end result is that, after replacing $b$ by something interdefinable with it (which we can do), we may assume that $S$ is a compact analytic subspace of $D(X)$ and that $Z$ is the associated subspace of $X \times S$. By Fact 2.1 (working with Douady spaces), the canonical morphism $f: Z \rightarrow X$ is Moishezon. By 2.2, $\operatorname{tp}(b, c / c)$ is Moishezon, and thus $\operatorname{tp}(b / c)$ is Moishezon.

REMARK 2.4. Let $b, c$ be as in the above theorem. Then for any tuple a from $\mathcal{A}^{*}, \operatorname{tp}(b / c a)$ is Moishezon.

Proof. The conclusion of Theorem 2.3 tells us that there is some set $C$ of parameters containing $c$ and such that $b$ is independent of $C$ over $c$ and $b \in \operatorname{dcl}(C, d)$ for some tuple $d$ from $\mathbb{C}^{*}$. Without loss of generality (that is, by automorphism) $C$ is independent of $a$ over $b c$, so independent of $b c a$ over $c$, and so independent of $b$ over $c a$.

Let us now give some applications. 
COROLlary 2.5. Let $p(x)$ be a stationary type of U-rank 1 over some set in $\mathcal{A}^{*}$. Then $p$ is either modular or nonorthogonal to (the generic type of ) $\mathbb{C}^{*}$.

Proof. As $\mathcal{A}^{*}$ has finite Morley rank (sort-by-sort) we may assume that $p(x)$ is over a finite tuple $a$ of parameters. If $p$ were not modular then by 2.2.6 of [12] there would be a tuple $c$ of realizations of $p$ (in fact a pair is sufficient) and some tuple $b$ such that $b=\mathrm{Cb}(\operatorname{tp}(c / b a))$ and $b \notin \operatorname{acl}(c a)$. By Remark 2.4, $\operatorname{tp}(b / c a)$ is Moishezon. In particular $\operatorname{tp}(b / c a)$ is nonorthogonal to the generic type of $\mathbb{C}^{*}$. But $b$ lives in $p^{\text {eq }}$, and thus $p$ is nonorthogonal to $\mathbb{C}^{*}$.

The next application concerns definable subsets of groups and homogeneous spaces. Definable groups in $\mathcal{A}$ have naturally the structure of "meromorphic groups" (see [13] and [8]). A definable group in $\mathcal{A}^{*}$ can be considered as the generic fibre of a meromorphic family of meromorphic groups.

Let us start with a general lemma about stable groups.

Lemma 2.6. Let $G$ be a connected group (type)-definable in a saturated stable structure $\bar{M}$. Let $c \in G$ be such that $p(x)=\operatorname{tp}(c)$ is stationary. Let $H$ be the left stabilizer of $p$. Let $a \in G$ be generic over $c$. Let $c / H$ denote $H c$ as an element of the right coset space $H \backslash G$. Then $c / H$ is interdefinable over a with $\operatorname{Cb}(\operatorname{tp}(a / c a))$. (Similarly with left and right interchanged.)

Proof. So $H \backslash G$ denotes the space $\{H g: g \in G\}$ of right cosets of $H$ in $G$, and as above we write $H g$ as $g / H$ when we want to treat it as an element rather than a definable set. Some more notation: given a stationary type $q$ over $\emptyset$ of some element of $G$, and given $a \in G$, by $q a$ we mean the restriction to $a$ of the translate $q^{\prime} a$ of $q^{\prime}$ by $a$ where $q^{\prime}$ is the global nonforking extension of $q$. Note that $q a$ is stationary and $q^{\prime} a$ is its global nonforking extension. Moreover $q a=\operatorname{tp}(d a / a)$ for $d$ realizing $q \mid a$.

Now let us make some observations.

$$
a \text { realizes } p^{-1} c a \text {. }
$$

This is because $c^{-1}$ realizes $p^{-1}$, and (as $a$ is generic in $G$ over $c$ ), $c a$ is generic over $c^{-1}$ so independent of $c^{-1}$.

(ii) $c a / H$ is interdefinable with $\mathrm{Cb}\left(p^{-1} c a\right)$.

Indeed, $H$ is the left stabilizer of $p$ so the right stabilizer of $p^{-1}$, which clearly yields (ii): an automorphism $f$ fixes $c a / H$ iff $p^{-1} c a$ is parallel to $f\left(p^{-1} c a\right)=p^{-1} f(c a)$.

Note that $c / H$ and $c a / H$ are interdefinable over $a$. So by (i) and (ii) we deduce that $c / H$ and $\operatorname{Cb}(\operatorname{tp}(a / c a))$ are interdefinable over $a$.

REMARK 2.7. Note that Lemma 2.6 includes the old result [5] that in modular (or 1-based) groups any stationary type is a translate of the generic 
type of a subgroup: if $\operatorname{Cb}(\operatorname{tp}(a / c a)) \in \operatorname{acl}(a)$ then $c / H \in \operatorname{acl}(\emptyset)$ and $\operatorname{sotp}(c)$ is a translate of the generic type of $H$.

Lemma 2.6 applies to groups definable in $\mathcal{A}^{*}$. So together with Theorem 2.3 we obtain:

Corollary 2.8. Let $G$ be a group definable in $\mathcal{A}^{*}$. Work over some algebraically closed set of parameters $A$ over which $G$ is definable. Let $c \in G$ and let $H$ be the left stabilizer of $\operatorname{tp}(c / A)$. Then $\operatorname{tp}((c / H) / A)$ is Moishezon.

For groups definable in $\mathcal{A}$, that is, meromorphic groups, Corollary 2.9 below gives a more geometric looking statement. As a matter of notation, if $X$ and $Y$ are complex (not necessarily compact) spaces definable in $\mathcal{A}$, we will say that $X$ and $Y$ are meromorphically isomorphic if there is a bihomolorphic map between $X$ and $Y$ which is also definable in $\mathcal{A}$.

COROLlaRY 2.9. Let $G$ be an arbitrary meromorphic group (not necessarily commutative). Let $X$ be an irreducible meromorphic subvariety of $G$, and let $H=\{g \in G: g \cdot X=X\}$ be the set-theoretic left stabilizer of $X$ in $G$. Let $\overline{H \backslash X}$ denote the Zariski closure of $H \backslash X$ in the meromorphic homogeneous space $H \backslash G$ of right cosets $H g(g \in G)$. Then $\overline{H \backslash X}$ is meromorphically isomorphic to an algebraic variety. (Likewise with left and right interchanged.)

Proof. Let $p=\operatorname{tp}(c / \mathcal{A})$ be the generic type of $X$. (So $c \in X^{*} \subseteq G^{*}$.) Then $H$ (or rather its extension $H^{*}$ ) as defined in the statement to be proved identifies with the left stabilizer of $p$. By Corollary 2.8, $\operatorname{tp}\left(\left(c / H^{*}\right) / \mathcal{A}\right)$ is Moishezon. As $\mathcal{A}$ is a model, this is witnessed over $\mathcal{A}$, namely $c / H^{*} \in \operatorname{dcl}(d)$ for some tuple from $\mathbb{C}^{*}$. It follows that $c / H^{*}$ is interdefinable with some tuple in $\mathbb{C}^{*}$. But $c / H^{*}$ is a generic point over $\mathcal{A}$ of $(\overline{H \backslash X})^{*} \subseteq(H \backslash G)^{*}$. As $H \backslash G$ is a homogeneous space it follows that $\overline{H \backslash X}$ is meromorphically isomorphic to an algebraic variety.

\section{References}

[1] F. Campana, Algébricité et compacité dans l'espace des cycles d'un espace analytique complexe, Math. Ann. 251 (1980), 7-18.

[2] F. Campana and T. Peternell, Cycle spaces, in [4], 319-349.

[3] A. Fujiki, On the Douady space of a compact complex space in the category $\mathcal{A}$, Nagoya Math. J. 85 (1982), 189-211.

[4] H. Grauert, T. Peternell and R. Remmert (eds.), Several Complex Variables VII, Springer, 1994.

[5] E. Hrushovski and A. Pillay, Weakly normal groups, in: Logic Colloquium '85, North-Holland, 1987, 233-244.

[6] E. Hrushovski and B. Zilber, Zariski geometries, Bull. Amer. Math. Soc. 28 (1993), $315-323$. 
[7] E. Hrushovski and B. Zilber, Zariski geometries, J. Amer. Math. Soc. 9 (1996), $1-56$.

[8] P. Kowalski and A. Pillay, Subvarieties of commutative meromorphic groups, preprint, 2001.

[9] R. Moosa, Saturated compact complex spaces, preprint, 2001.

[10] - Contributions to the model theory of fields and compact complex spaces, Ph.D. thesis, Univ. of Illinois, 2001.

[11] A. Pillay, Some model theory of compact complex spaces, in: Hilbert's 10th Problem, Contemp. Math. 270, Amer. Math. Soc., 2000, 323-338.

[12] A. Pillay, Geometric Stability Theory, Oxford Univ. Press, 1996.

[13] A. Pillay and T. Scanlon, Meromorphic groups, preprint, 2000.

[14] A. Pillay and M. Ziegler, Jet spaces of varieties over differential and difference fields I, preprint, 2001.

[15] K. Ueno, Classification Theory of Algebraic Varieties and Compact Complex Spaces, Lecture Notes in Math. 439, Springer, 1975.

Department of Mathematics

University of Illinois at Urbana-Champaign

Altgeld Hall

1409 W. Green St.

Urbana, IL 61801, U.S.A.

E-mail: pillay@math.uiuc.edu
Institut für Mathematik Humboldt Universität D-10099 Berlin, Germany

Received 15 November 2001; in revised form 19 March 2002 